\title{
LOS IMPACTOS DEL TURISMO EN ESPAÑA: DIFERENCIAS ENTRE DESTINOS DE SOL Y PLAYA Y DESTINOS DE INTERIOR
}

\author{
Diana Gómez Bruna \\ Clara Martín Duque \\ Universidad Antonio de Nebrija. Madrid
}

\section{RESUMEN}

El presente artículo tiene como objetivo la evaluación de los impactos económicos, socioculturales y medioambientales ocasionados por el turismo en España. En concreto, el artículo mide la percepción que de ellos tienen los residentes de 151 municipios turísticos valorando las posibles diferencias entre las percepciones de los residentes de destinos de sol y playa y de interior. Para ello, se han realizado 1027 encuestas analizadas a través de la prueba paramétrica para la comparación de medias (ANOVA). Este análisis ha permitido determinar, entre otras cuestiones, que los residentes de los destinos de sol y playa perciben los impactos del turismo de forma más negativa que los de los destinos de interior.

Palabras clave: impactos, residentes, turismo, destinos turísticos.

Tourism impacts in Spain: differences between hinterlands and coastal areas

\section{ABSTRACT}

This article aims to evaluate the perception of the Spanish residents about the economic, sociocultural and environmental impacts that tourism generate in Spain. In particular, the article analyse the resident percepcion of the impacts in 151 spanish destinations making distinctions between coastal destinations and hinterland destinations. To this end we have made a one way ANOVA based on 1027 surveys. This analysis of the data shows that those resident who live in coastal areas perceive more strongly the negatives effects of tourism than those who live in hinterlands.

Keywords: impacts, residents, tourism, tourist destination.

Fecha de recepción: 11 de octubre de 2017

Fecha de aceptación: 16 de julio de 2018

Departamento de Turismo. Universidad Antonio de Nebrija. C/Santa Cruz de Marcenado, 27

28015 MADRID (España).E-mail: dgomezb@nebrija.es, cmartidu@nebrija.es 


\section{INTRODUCCIÓN}

Uno de los ámbitos de estudio más analizados en la literatura relacionada con los destinos turísticos versa sobre los impactos generados por el turismo y las percepciones que de ellos tienen los grupos de interés involucrados, muy especialmente la población local.

Desde el punto de vista práctico, hasta principios de los setenta la mayoría de los estudios realizados en torno a la medición de los impactos del turismo se centraron en los impactos económicos. Este hecho se debe, en cierto modo, a la visión optimista que en esos años se tenía del turismo, así como a la mayor facilidad para la cuantificación de estos impactos frente a los impactos sociales o medioambientales (Wall y Mathieson, 2006).

Sin embargo, ya en esos años, empiezan a aparecer algunos estudios con una visión menos optimista que se centran en los efectos psicológicos, sociales y culturales del turismo, cuyos precursores (Young, 1973; Turner y Ash, 1975) fueron seguidos de muchos otros (Graburn 1976; Smith 1977; Cohen 1978; Pizam 1978) que comenzaron a enfocar sus análisis en el estudio de la prevención de los efectos negativos del turismo.

Desde entonces, un buen número de autores se ha centrado en estudiar las percepciones y actitudes hacia el turismo de los residentes de los destinos como elemento clave del éxito y la sostenibilidad turística (Garau-Vadell, Gutiérrez-Taño y Díaz-Armas, 2018). La mayoría de estos estudios se han centrado en analizar los diversos factores que influyen en dicha percepción de los impactos, tales como las características sociodemográficas de los grupos de interés (Williams y Lawson, 2001; Sharma y Dyer, 2009), la proximidad de la residencia a los centros turísticos (Fredline y Faulkner, 2000; Jurowski y Gursoy, 2004; Daniels, 2007), el grado de contacto con los turistas (Ap, 1992; Lankford, 1994; Akis, Peristianis y Warner, 1996; Andereck, Valentine, Vogt y Knopf, 2007), el nivel de desarrollo turístico del destino (Doxey, 1976; Pearce, 1980; Long, Perdue y Allen, 1990) o la situación de la economía (Garau-Vadell et al., 2018).

A pesar de la abundante literatura, hay escasos estudios (Sharma y Dyer, 2009; Markovic y Klaric, 2015; Rasoolimanesh, Ringle, Jaafar y Ramayahm 2017) que, sin embargo, hayan comparado las percepciones que los residentes tienen de los impactos económicos, socioculturales y medioambientales generados por el turismo en función de la tipología de destino. La escasez de investigaciones previas supone un reto para la presente investigación que ha establecido como objetivo fundamental analizar si existen diferencias significativas entre las percepciones de los residentes de los destinos turísticos españoles en función del tipo de destino: sol y playa frente a destinos de interior.

\section{REVISIÓN TEÓRICA DEL UNIVERSO TIPOLÓGICO DE LOS IMPACTOS TURÍSTICOS}

El modelo de sostenibilidad turística se constituye como un paradigma ideal de equilibrio entre "la preservación del patrimonio natural y cultural, la viabilidad económica del turismo y la equidad social del desarrollo" (Ivars, 2001:9).

Este equilibrio es complejo ya que la naturaleza, incluso sin la intervención humana, experimenta de forma normal un continuo flujo de cambios que tienen un ritmo determinado. El problema se produce cuando el ser humano interviene de forma agresiva en el 
entorno y acelera esos cambios (Wall y Mathieson, 2006) comprometiendo los procesos y factores económicos, sociales y medioambientales (Mason, 2003). Dentro de todos los cambios que se producen en el entorno, es necesario diferenciar entre aquellos que se generan de manera natural y aquellos inducidos por el hombre si bien, esta diferenciación resulta compleja dado que es difícil remontarse hasta el momento en que la naturaleza se encontraba en un estado primitivo (Wall y Mathieson, 2006).

En este sentido, la actividad turística se erige como una posible amenaza frente a un paradigmático equilibrio en los destinos, que se comportan, en definitiva, como pequeños ecosistemas susceptibles de experimentar mutaciones cuando una actividad o personas ajenas al mismo entran en contacto con ellos (Wall y Mathieson, 2006).

Estas mutaciones o cambios provocados por el turismo son lo que se ha denominado "los impactos del turismo"1, normalmente relacionados tanto con aspectos negativos como positivos pues los impactos pueden ser identificados como una amenaza para el destino pero también como una oportunidad (Mason, 2003; Lázaro, 2004; Wall y Mathieson, 2006). En cuanto a su universo tipológico, los impactos generados por el turismo se han clasificado en impactos económicos, socioculturales y medioambientales (Wall y Mathieson, 2006).

En general los impactos positivos se han tendido a relacionar con los impactos económicos (Tosun, 2002; Wall y Mathieson, 2006; Vázquez Varela y Martín Gil, 2011) pues, el turismo, es uno de los sectores con más capacidad para dinamizar las economías locales (Sancho, 1998). Entre los impactos económicos positivos más mencionados en la literatura encontramos: la creación de empleo (Pizam, 1996; Lindberg y Johnson, 1997; Figuerola, 2000; Coccossis y Mexa, 2002; OCDE, 2002; Tosun, 2002; Mason, 2009; Tribe, 2009; World Travel \& Tourism Council, 2013); el aumento de los ingresos por turismo (Figuerola 2000; Tribe, 2009); la generación de ingresos fiscales directos e indirectos (Figuerola, 2000;Coccossis y Mexa, 2002; Tribe, 2009); la contribución al equilibrio de los saldos de la balanza de pagos (Sancho, 1998; Figuerola, 2000; Tribe, 2009; World Travel \& Tourism Council, 2013); el desarrollo intersectorial o efecto multiplicador del turismo (Sancho, 1998; Figuerola 2000; Coccossis y Mexa, 2002; OMT, 2005); la redistribución de la renta (Sancho, 1998; Figuerola, 2000; OCDE, 2002); el aumento de las inversiones o la influencia en la formación bruta de capital fijo (Figuerola, 2000; World Travel \& Tourism Council, 2013).

También existe un buen número de autores que han apostado por los impactos positivos que genera la actividad turística en el ámbito medioambiental. Entre ellos destacan la mayor inversión en conservación de áreas tanto naturales como históricas (Liu, Sheldon y Var, 1987; Akis et al., 1996; Yoon, Gursoy y Chen, 2001; Coccossis y Mexa, 2002; Andereck et al., 2005; Holden, 2009; Mason, 2009); el desarrollo de acciones para mejorar el entorno de las áreas turísticas (Sancho, 1998; Coccossis y Mexa, 2002; Garau-Vadell et al., 2018); la mejora de las infraestructuras locales (Coccossis y Mexa, 2002); la generación de estándares de calidad para certificar el buen estado del medio ambiente (Sancho, 1998);

1 Se entiende como impactos "Efectos de largo plazo positivos y negativos, primarios y secundarios, producidos directa o indirectamente por una intervención para el desarrollo, intencionalmente o no" (OCDE, 2010: 24). 
o el incremento de la preocupación por el medio ambiente por parte de la población local (Coccossis y Mexa, 2002).

\section{Tabla 1}

\section{REVISIÓN TEÓRICA DE LOS IMPACTOS POSITIVOS GENERADOS EN EL TURISMO}

\begin{tabular}{|l|l|}
\hline \multirow{3}{*}{ Impactos económicos positivos } & $\begin{array}{l}\text { Pizam (1996); Lindberg y Johnson (1997); Sancho (1998); } \\
\text { Figuerola (2000); Coccossis y Mexa (2002); OCDE (2002); } \\
\text { Tosun (2002); OMT (2005); Wall y Mathieson (2006); Mason } \\
\text { (2009); Tribe (2009); Vázquez Varela y Martín Gil (2011); } \\
\text { World Travel \& Tourism Council (2013) }\end{array}$ \\
\hline $\begin{array}{l}\text { Impactos medioambientales } \\
\text { positivos }\end{array}$ & $\begin{array}{l}\text { Liu, Sheldon y Var (1987); Akis } \text { et al. (1996); Sancho (1998); } \\
\text { Gursoy y Chen (2001); Yoon } \text { et al., (2001); Coccossis y Mexa } \\
\text { (2002); Andereck } \text { et al., (2005); Holden (2009); Mason (2009); } \\
\text { Garau-Vadell } \text { et al. (2018) }\end{array}$ \\
\hline $\begin{array}{l}\text { Impactos socioculturales } \\
\text { positivos }\end{array}$ & $\begin{array}{l}\text { Yoon et al. (2001); Besculides, Lee y McCormick (2002); } \\
\text { Coccossis y Mexa (2002); Andereck et al. (2005); OMT } \\
\text { (2005); Pereiro y De León (2007); Royo y Ruiz (2009); Royo } \\
\text { y Ruiz (2009); Mason (2009) }\end{array}$ \\
\hline
\end{tabular}

Fuente: Elaboración propia.

Por último, también se han identificado en la literatura académica diversos impactos positivos socioculturales. Entre los más importantes destacan el intercambio cultural y la preservación de la identidad cultural (Andereck et al., 2005; Besculides, Lee y McCormick, 2002; Yoon et al., 2001; Coccossis y Mexa, 2002; Pereiro y De León, 2007; Royo y Ruiz, 2009). En esta misma línea destacan también el aumento de las actividades de ocio para los residentes (OMT, 2005; Royo y Ruiz, 2009); la revitalización de las artes tradicionales y lenguas (OMT, 2005; Mason, 2009); la ruptura de los estereotipos negativos (OMT, 2005; Royo y Ruiz, 2009); la disminución de desigualdades sociales (OMT, 2005); la mejora de la conservación de recursos culturales de especial interés turístico (Mason, 2009) o la mejora de las condiciones sanitarias o educativas (Coccossis y Mexa, 2002; OMT, 2005; Pereiro y De León, 2007).

A pesar de esta visión optimista de la actividad turística también es cierto que, como mencionábamos anteriormente, desde los años 70 el desarrollo turístico ha generado un gran debate porque desde esa época se han tratado de identificar y analizar los impactos negativos que genera el turismo tanto en lo económico como en lo medioambiental o sociocultural.

En cuanto a los perjuicios que puede provocar el turismo en las economías locales, la literatura académica ha resaltado impactos como el aumento de la presión inflacionaria del turismo (Figuerola, 2000; Coccossis y Mexa, 2002); el incremento de la especulación sobre el valor del suelo (Figuerola, 2000; Hernández, 2004); la excesiva dependencia del 
turismo en algunos destinos (Coccossis y Mexa, 2002; OMT 2005) o la desigual distribución de los beneficios económicos (Coccossis y Mexa, 2002; OMT, 2005).

Sin embargo, el área donde estos impactos negativos han sido más visibles ha sido la medioambiental por tratarse de impactos directos sobre el medioambiente físico (OMT, 2005; Holden, 2009). Entre ellos destacan la pérdida de la biodiversidad (Coccossis y Mexa, 2002; OCDE, 2002; Pérez, 2004; OMT, 2005; Garu-Vadell et al., 2018); la generación de contaminación acústica (Coccossis y Mexa, 2002; OCDE, 2002; Pérez, 2004; Pulido, 2004; OMT 2005; Holden, 2009); la contribución a la contaminación de las aguas (Coccossis y Mexa, 2002; OCDE, 2002; Pérez, 2004; Pulido, 2004; OMT 2005; Holden, 2009); la mayor erosión del suelo (Sancho, 1998; OCDE, 2002; OMT 2005); la contaminación del aire por la emisión de gases de efecto invernadero y contribución al cambio climático (Coccossis y Mexa, 2002; OCDE, 2002; Pérez, 2004; Pulido, 2004; OMT 2005; Almeida-García, Peláez-Fernández, Balbuena-Vázquez y Cortés-Macias, 2016); la contribución a la contaminación del paisaje o contaminación visual (Sancho, 1998; Coccossis y Mexa, 2002; OCDE, 2002; Pérez, 2004; OMT 2005; Holden, 2009); la generación de masificación en algunas zonas que degrada la calidad de la visita y dificulta la conservación de los recursos (Coccossis y Mexa, 2002; Pérez, 2004; OMT 2005); el aumento del gasto de recursos escasos como el agua o la electricidad (Coccossis y Mexa, 2002; Pulido, 2004; OMT 2005) o la ocupación de suelo valioso por ocupciones turísticas (Pulido, 2004).

Tabla 2

\section{REVISIÓN TEÓRICA DE LOS IMPACTOS NEGATIVOS GENERADOS EN EL TURISMO}

\begin{tabular}{|l|l|}
\hline $\begin{array}{l}\text { Impactos económicos } \\
\text { negativos }\end{array}$ & $\begin{array}{l}\text { Figuerola, (2000); Coccossis y Mexa (2002); Hernández (2004); } \\
\text { OMT (2005) }\end{array}$ \\
\hline $\begin{array}{l}\text { Impactos medioambientales } \\
\text { negativos }\end{array}$ & $\begin{array}{l}\text { Sancho (1998); Coccossis y Mexa (2002); OCDE (2002); Pérez } \\
\text { (2004); Pulido (2004); OMT (2005); Holden (2009) }\end{array}$ \\
\hline $\begin{array}{l}\text { Impactos socioculturales } \\
\text { negativos }\end{array}$ & $\begin{array}{l}\text { Haralambopoulos y Pizam (1996); Sancho (1998); Coccossis y } \\
\text { Mexa (2002); OMT (2005); Pereiro y De León (2007); Diedrich } \\
\text { al. (2015) }\end{array}$ \\
\hline
\end{tabular}

Fuente: Elaboración propia.

Por último, en los últimos años es cuando se ha prestado una mayor atención a los impactos negativos que genera el turismo desde el punto de vista sociocultural. Entre ellos destacan el cambio en las actividades tradicionales por influencia externa (Coccossis y Mexa, 2002; OMT, 2005; Figuerola et al., 2015); la invasión de la privacidad y el desplazamiento de las actividades tradicionales (Coccossis y Mexa, 2002; OMT, 2005); la generación del "efecto demostración" sobre los residentes (Sancho, 1998; Mason, 2009); el aumento de las 
desigualdades y conflictos por el desigual acceso y uso de recursos así como por la distribución de los beneficios (Sancho, 1998; Pereiro y De León, 2007); la mayor tensión en la comunidad por la presencia de personas ajenas a la misma (Coccossis y Mexa, 2002; OMT, 2005; Royo y Ruiz, 2009); la aculturación (Coccossis y Mexa, 2002; OMT, 2005; Mason, 2009); la pérdida de acceso a las actividades de recreo y ocio para los residentes en favor de los turistas (Coccossis y Mexa, 2002; OMT, 2005); el aumento de la congestión de personas y dificultad para acceder a infraestructuras básicas o recursos del destino (Coccossis y Mexa, 2002; OMT, 2005) o el aumento de problemas sociales: prostitución, criminalidad, etc. (Haralambopoulos y Pizam, 1996; OMT, 2005 Diedrich y García, 2009).

En este contexto, la medición de los impactos turísticos se convierte en una herramienta imprescindible para la existencia de una relación armónica entre turismo y sostenibilidad (Lázaro, 2004). Tal y como indica Wu (2009), más allá de la teoría, para tratar de alcanzar la sostenibilidad se debe tratar de entender los impactos tanto económicos como sociales y medioambientales que el turismo puede causar en el destino, así como las dinámicas que se establecen entre estos impactos. Es decir, la idea de turismo sostenible implica "el reconocimiento de los impactos negativos y la necesidad de gestionarlos para alcanzar los objetivos de desarrollo sostenible" (Saarien, 2006:1126).

\section{LA MEDICIÓN DE LOS IMPACTOS TURÍSTICOS: LAS PERCEPCIONES DE LOS RESIDENTES EN DESTINOS TURÍSTICOS}

Los impactos del turismo evolucionan y se transforman en un sistema denominado por Liburd y Edwards (2010) como adaptativo, es decir, como un sistema con dinámicas no lineales que pueden producir cambios muy difíciles de predecir. A pesar de esta dificultad, identificar los impactos y tener capacidad de respuesta frente a ellos puede ayudar al destino a ser más fuerte frente a las amenazas que pueden desequilibrar la sostenibilidad del territorio.

Entre las herramientas más utilizadas en la actualidad para medir los impactos del turismo se encuentran los indicadores de sostenibilidad que ayudan a identificar las necesidades y proporcionan información comparable de los destinos ${ }^{2}$. El problema de este sistema radica en que normalmente se diseñan una serie de indicadores estándar que no captan la complejidad de la actividad turística (Sancho y García, 2006).

Asimismo, en general, los estudios en turismo han priorizado la utilización de indicadores objetivos, otorgando menos importancia a los indicadores subjetivos, es decir, a aquellos destinados a medir las percepciones o actitudes de los agentes del destino, muy especialmente de la población local (Sancho, García y Rozo, 2007).

Sin embargo, a partir de los años 70 el análisis de estas percepciones se ha convertido en un área de estudio fundamental (Pizam, 1978; Thomason, Crompton, y Kamp, 1979; Liu y Var, 1986; Long, Perdue, y Allen, 1990; Lankford, 1994; Jurowski y Gursoy, 2004; Vargas-Sánchez, Plaza-Mejía y Porras-Bueno, 2009; Almeida-García et al., 2016) por

2 Se entiende como indicador de sostenibilidad " aquellas medidas cualitativas y cuantitativas que permiten conocer, cuando se aplican periódicamente los cambios producidos, a favor o en contra, en relación con las condiciones ambientales, socioculturales o económicas de un destino turístico con el fin de tomar decisiones estratégicas de mejoramiento, mantenimiento o transformación de una situación dada" (Sancho et al., 2007:9) 
las repercusiones que dichas percepciones pueden tener en el éxito del destino turístico. En concreto, un buen número de autores consideran que percepciones negativas pueden afectar a la sostenibilidad del destino (Butler, 1980; Ap, 1992; Jurowski y Gursoy, 2004; Diedrich y García, 2009; Garau-Vadell et al., 2018); a las relaciones entre los residentes y los turistas (Pearce, 1998; Gursoy, Jurowski, y Uysal, 2002); o a la capacidad emprendedora en el destino (Garau-Vadell et al., 2018).

Así pues, las opiniones de los residentes y su apoyo al turismo se convierten en un factor clave en el desarrollo turístico (Butler, 1980; Dyer, Gursoy, Sharma y Carter, 2007). De este modo, los estudios al respecto apuntan a la necesidad de analizar los impactos no solamente a través de indicadores objetivos sino también de indicadores subjetivos como es el análisis de percepción ${ }^{3}$ que de ellos tienen los residentes de los destinos. Tal y como afirman McGehee y Anderek (2004: 132) "entender las percepciones que los residentes tienen de los impactos del turismo es tan importante, si no más, que entender los impactos mismos". En definitiva, identificar y evaluar tales percepciones puede contribuir a minimizar los impactos negativos del turismo y maximizar los positivos (Vargas-Sánchez, Porras-Bueno y Plaza-Mejía, 2011).

En este sentido, tal ha sido la proliferación de literatura al respecto que es complejo hacer una revisión completa de toda ella, si bien, algunos autores han realizado interesantes intentos (Easterling, 2004; Deery, Jago y Fredline, 2012; Sharpley, 2014). De ellas podemos extraer que, en general, los estudios sobre percepciones de los residentes pueden ser clasificados según las metodologías utilizadas, la localización del estudio o las variables de influencia sobre las percepciones hacia el turismo.

En cuanto a las metodologías utilizadas, cabe destacar una gran presencia de metodologías cuantitativas (Sharpley, 2014) que en general utilizan la técnica de la encuesta analizando los resultados a través de análisis de la varianza (Ritchie, 1988; King, Pizam y Milman 1993; Andereck et al., 2005; Sharma y Dyer, 2009; Vargas-Sánchez et al., 2011); análisis factorial (Long, Perdue y Allen, 1990; Haralambopoulos y Pizam, 1996; Tosun, 2002; Andereck et al., 2005; Andereck y Nyaupane, 2011; Lundberg, 2017); análisis cluster (Madrigal, 1995; Fredline y Faulkner, 2000; Aguiló y Roselló, 2005) o modelos de ecuaciones estructurales (Lindberg y Johnson, 1997; Gursoy et al., 2002; Gursoy et al., 2010; Choi y Murray, 2010; Cardona, 2012).

Por otro lado, una revisión más profunda de la literatura nos muestra el desequilibrio en cuanto a zonas de estudio analizadas. El mundo desarrollado es sin duda el que más interés ha suscitado, siendo el Norte América la región más analizadas seguida de otras regiones como Nueva Zelanda o Reino Unido y dejando de lado otras regiones mundiales con un gran desarrollo turístico como Caribe y el Mediterráneo (Sharpley, 2014)

En concreto, a pesar de que España es uno de los destinos turísticos más importantes a nivel internacional, no existe una amplia literatura que haya analizado la percepción de los residentes hacia el turismo. Solo en la última década han aparecido estudios que, en general, han analizado destinos o zonas concretas de la geografía española: Marrero (2006) y Gutiérrez (2010) en las Islas Canarias; Aguiló, García y Rosello (2004); Pérez y Nadal (2005); Bujosa y Rossello (2007) y Cardona (2012) en las Islas Baleares; Royo y Ruíz

3 El término percepción es ampliamente utilizado si bien, algunos atores utilizan el de actitud (Gursoy et al., 2002; Lindberg y Johnson, 1997) o el de reacción (Fredline y Faulkner, 2000). 
(2009) en Cataluña; Huete (2010) en Valencia; Vargas et al. (2009 y 2011) en Huelva; Oviedo et al. (2008) en Sevilla y Almeida-García et al., (2016) en Málaga.

Finalmente, la literatura muestra el interés de los diversos autores por conocer cuáles son las variables de influencia sobre las percepciones del turismo ya que estas resultan un elemento clave para comprender las posibles diferencias entre las percepciones que del turismo tienen unos u otros individuos (Akis et al., 1996).

\subsection{Variables que influyen en la percepción de los impactos turísticos}

A la hora de identificar y medir la percepción de los impactos que tienen los residentes del destino, resulta fundamental conocer aquellas variables que pueden influir en tales percepciones. En este sentido, a pesar de la existencia de múltiples clasificaciones, la que parece más aceptada en la literatura académica es la que distingue entre variables intrínsecas (relacionadas con el individuo) y extrínsecas (externas al individuo) (Faulkner y Tideswell, 1997; Sharpley, 2014; Garau-Vadell et al., 2018).

\section{Tabla 3}

\section{VARIABLES DE INFLUENCIA EN LA PERCEPCIÓN DE LOS RESIDENTES SOBRE EL TURISMO}

\begin{tabular}{|c|c|}
\hline \multicolumn{2}{|l|}{ Variables Intrínsecas } \\
\hline $\begin{array}{l}\text { Demográficas: edad, género, } \\
\text { educación, etc. }\end{array}$ & $\begin{array}{l}\text { Haralambopoulos y Pizam (1996), Fredline y Faulkner (2000), } \\
\text { Williams y Lawson (2001), Sharma y Dyer (2009), Almeida- } \\
\text { García et al. (2016) }\end{array}$ \\
\hline Grado de contacto con los turistas & $\begin{array}{l}\text { Ap (1992), Lankford (1994), Akis et al. (1996), Teye, Sönmez } \\
\text { and Sirakaya (2002), Andereck et al. (2005) }\end{array}$ \\
\hline $\begin{array}{l}\text { Proximidad de la residencia a los } \\
\text { centros turísticos }\end{array}$ & $\begin{array}{l}\text { Fredline y Faulkner (2000), Jurowski y Gursoy (2004), Sharma } \\
\text { y Dyer (2009) }\end{array}$ \\
\hline Apego a la comunidad & $\begin{array}{l}\text { Sheldon y Var (1984), Gursoy et al. (2002) Andereck et al. } \\
\text { (2005) }\end{array}$ \\
\hline \multicolumn{2}{|l|}{ Variables Extrínsecas } \\
\hline Destino donde tienen lugar & $\begin{array}{l}\text { Wall y Mathieson (2006), Royo y Ruiz (2009), Díaz y Gutiérrez } \\
\text { (2010) }\end{array}$ \\
\hline $\begin{array}{l}\text { Nivel de desarrollo turístico del } \\
\text { destino }\end{array}$ & $\begin{array}{l}\text { Doxey (1976), Pearce (1980), Long et al. (1990), Holden } \\
\text { (2009), Vargas-Sánchez et al. (2009) }\end{array}$ \\
\hline $\begin{array}{l}\text { Densidad del desarrollo turístico } \\
\text { / turistas }\end{array}$ & $\begin{array}{l}\text { Bestard y Nadal (2007), Holden (2009), Vargas-Sánchez et al. } \\
\text { (2011) }\end{array}$ \\
\hline Tipología de destino & $\begin{array}{l}\text { Sharma y Dyer (2009), Markovic y Klovic (2015), } \\
\text { Rasoolimanesh et al. (2017) }\end{array}$ \\
\hline Características de los turistas & $\begin{array}{l}\text { Sheldon y Var (1984), Johnson et al. (1994), Mason (2009), } \\
\text { Royo y Ruiz (2009) }\end{array}$ \\
\hline Estacionalidad & Sheldon y Var (1984), Mason (2003) \\
\hline Situación de la economía local & Garau-Vadell et al.(2018) \\
\hline
\end{tabular}

Fuente: Elaboración propia. 
Como muestra la tabla 3, son numerosas las variables estudiadas si bien, los resultados de los diversos estudios analizados son en ocasiones contradictorios y no permiten identificar de manera clara los factores fundamentales que determinan las percepciones que los residentes tienen del turismo.

Por otro lado, tras la revisión de la literatura académica se ha detectado que hay escasos estudios (Sharma y Dyer, 2009; Markovic y Klaric, 2015; Rasoolimanesh et al., 2017) que hayan analizado diferencias de las percepciones de los residentes en función del tipo de destino en el que residen. En este sentido, en esta investigación nos hemos centrado precisamente en las características del destino turístico (destinos de sol y playa o destinos de interior) como una de las variables que puede influir en la distinta percepción de los impactos por parte de los residentes para el caso español.

A este respecto, debemos indicar que si bien no existen demasiadas investigaciones que aborden esta cuestión, algunos autores (Rodríguez, 1994; Zahedi, 2008) se atreven a relacionar el turismo masivo y más destructivo con los destinos turísticos de sol y playa, siendo aquellos impactos negativos relacionados con el medioambiente los que sufren con mayor intensidad. En el caso español, el modelo predominante en los destinos de sol y playa españoles más desarrollados (cuenca mediterránea) coinciden con esta descripción (Soares, Gandara e Ivars, 2012) por lo que podemos pensar que puede darse la misma situación.

En las investigaciones desarrolladas en relación al grado de desarrollo turístico del destino, los resultados no son concluyentes si bien, en general, se perciben percepciones más negativas cuanto mayor es el grado de desarrollo del destino (Doxey, 1975; Smith, 1977; Butler, 1980; Sheldon y Var, 1984; Long et al., 1990; Vargas-Sánchez et al., 2009). En esta misma línea se enmarcan los resultados de los estudios que miden la densidad de turistas (Vargas-Sánchez et al., 2011) ya que las conclusiones apuntan a que cuanto mayor densidad de turistas, más negativas son las percepciones que del turismo tienen los residentes.

Respecto a los estudios que sí que se han centrado en diferentes tipologías de destino, destaca el trabajo desarrollado por Sharma y Dyer (2009) sobre la Costa del Sol australiana donde aseguran que los impactos sociales del turismo son percibidos de forma más positiva en las localidades de interior, menos desarrolladas que en las zonas de costa, mientras que los impactos económicos del turismo son percibidos de mejor manera en las localidades de costa. Por su parte, en el estudio de Markovic y Klaric (2015) enmarcado en Croacia, los resultados indican que si bien el turismo se ve de forma general como una actividad positiva por los efectos que tiene en la economía, las regiones del interior lo perciben de forma más positiva que las regiones costeras. Por último, la investigación de Rasoolimanesh et al. (2017) analiza las diferencias en las percepciones de las zonas rurales y urbanas de una región de Malasia detectando que los residentes en áreas urbanas tienen una percepción más positiva de los impactos generados por el turismo que los que residen en áreas rurales.

Tal y como mencionamos al comienzo del artículo, el principal objetivo del mismo se dirige a conocer la percepción de los impactos económicos, socioculturales y medioambientales generados por el turismo por parte de los residentes de los destinos turísticos españoles analizando si existen diferencias en dichas percepciones en función del tipo de 
destino turístico: destinos de sol y playa frente a destinos de interior. Así, las hipótesis principales de la investigación son:

$\mathrm{H}_{1}$. Existen diferencias significativas en la percepción que los residentes tienen de los impactos positivos generados por el turismo en los destinos españoles de sol y playa y los de interior.

$\mathrm{H}_{2}$. Existen diferencias significativas en la percepción que los residentes tienen de los impactos negativos generados por el turismo en los destinos españoles de sol y playa y los de interior.

$\mathrm{H}_{3}$. Los residentes de destinos turísticos de interior perciben de forma más intensa los impactos positivos generados por el turismo.

$\mathrm{H}_{4}$. Los residentes de destinos turísticos de sol y playa perciben de forma más intensa los impactos negativos generados por el turismo.

\section{METODOLOGÍA}

Teniendo en cuenta el objetivo principal de nuestro estudio y las hipótesis planteadas, consideramos que el enfoque metodológico más apropiado era el cuantitativo y que la técnica adecuada es la encuesta ya que esta "utiliza la recolección y el análisis de datos para contestar preguntas de investigación y probar hipótesis establecidas previamente, y confía en la medición numérica, el conteo y frecuentemente en el uso de la estadística para establecer con exactitud patrones de comportamiento en una población" (Hernández, Fernández y Baptista, 2010:5).

Nuestro estudio empírico se centra en España por ser uno de los países líderes tanto en llegadas de turistas internacionales, 75,6 millones en 2016 (INE, 2017a) como en ingresos internacionales por turismo, 77.625 millones de euros en 2016 (INE, 2017b), convirtiendo al turismo en uno de los sectores estratégicos para el desarrollo socioeconómico desde que comenzara a desarrollarse el turismo a finales de los años 50.

Así, la población de nuestra investigación queda formada por todos los sujetos involucrados de manera directa o indirecta con el sistema turístico de los 151 puntos turísticos establecidos por la Encuesta de Ocupación Hotelera (EOH) que elabora el Instituto Nacional de Estadística (INE).

Dada la inexistencia de una base de datos de los individuos objeto de nuestro estudio, se elabora una base de datos que finalmente está formada por 11.251 contactos y que permitió lograr una alta tasa de respuesta ya que finalmente se obtuvieron 1.027 respuestas válidas que suponían un error muestral del $2,91 \%$.

\subsection{Instrumento y procedimiento}

El diseño del instrumento de investigación, el cuestionario, se realiza con el objetivo de que las diversas cuestiones en él incluidas nos permitan cumplir con el objetivo general y los objetivos específicos establecidos para la investigación empírica.

El cuestionario recoge treinta preguntas cerradas estructuradas en dos bloques. El primero de ellos dedicado a conocer las características sociodemográficas principales de los encuestados, mientras que el segundo bloque se diseñó teniendo en cuenta la 
literatura previa (Gursoy y Rutherford, 2004) y está destinado a conocer la percepción de los encuestados acerca de los impactos económicos que el turismo genera en el destino en el que residen. En concreto, el instrumento de medida incluye 11 afirmaciones relacionadas con las tres grandes dimensiones de los impactos, económica, sociocultural y medioambiental. Los encuestados debían responder su grado de acuerdo con las afirmaciones en una escala de Likert (5 puntos) donde 1 era "Totalmente en desacuerdo" y 5 "Totalmente de acuerdo".

El cuestionario final se envía, junto con una carta de presentación en la que se justifican los motivos de la encuesta, a los 11.251 individuos que formaron nuestra base de datos.

Tabla 4

FICHA TÉCNICA DE LA ENCUESTA

\begin{tabular}{|l|l|}
\hline Población & $\begin{array}{l}\text { Infinita (todas las personas relacionadas con los 151 destinos } \\
\text { turísticos seleccionados) }\end{array}$ \\
\hline Población muestreada & Finita: 11.251 individuos \\
\hline Ámbito geográfico & España \\
\hline Tipo de entrevista & Encuesta autoadministrada por internet \\
\hline Procedimiento de muestreo & Aleatorio polietápico \\
\hline Tamaño muestral & $\mathrm{N}=1.027$ \\
\hline Error muestral & $\pm 2,91 \%$ (con $\mathrm{p}=\mathrm{q}=50$ y confianza del $95 \%)$ \\
\hline Fecha de trabajo de campo & Noviembre - Diciembre 2016 \\
\hline Tratamiento de la información & $\begin{array}{l}\text { Doble grabación de los datos en Excel } \\
\text { Paquete estadístico IBM SPSS (versión 20,0) }\end{array}$ \\
\hline
\end{tabular}

Fuente: Elaboración propia.

La distribución del cuestionario ha sido electrónica, vía e-mail. Finalmente, el cuestionario ha sido cumplimentado por un total de 1.027 contactos, determinando una muestra con un nivel de confianza del $95 \%$ y un error de $\pm 2,91 \%$ y $p=q=50$. Las respuestas obtenidas han sido procesadas con el software SPSS Versión 20.0 de IBM.

Los datos han sido analizados mediante un análisis univariante de tipo descriptivo seguido de un análisis inferencial bivariante a través de la prueba paramétrica para la comparación de medias (ANOVA) que nos ha permitido examinar las relaciones existentes entre la variable independiente (tipo de destino) y las variables dependientes relativas a la "percepción de los impactos de turismo ene le destino". En cuanto al tipo de destino hemos distinguido entre comunidades autónomas en las que predomina el turismo de sol y playa (Cataluña, Comunidad Valenciana, Murcia, Andalucía, Islas Baleares e Islas Canarias) y comunidades autónomas de interior. Con el objetivo de conocer las relaciones que se establecen entre dichas variables, se ha tomado el valor estadístico F de Fisher Snedecor aceptando valores $p$ por debajo de .05 como estadísticamente significativos. 


\subsection{Muestra}

La muestra está formada por un total de 1.027 cuestionarios cuyo perfil se corresponde, de manera mayoritaria, con edades comprendidas entre los 34 y los 56 años y con estudios universitarios; pertenecientes a las comunidades autónomas de Andalucía, Cataluña, Madrid, Comunidad Valenciana, Islas Baleares e Islas Canarias y que se identifican, mayoritariamente, con el sector privado, especialmente con el subsector de alojamientos, y con la sociedad civil, con asociaciones de vecinos y culturales.

La mayor parte de la muestra se concentra en unas edades comprendidas entre los 34-45 años y entre los 46-56 años mientras la distribución geográfica de las respuestas se concentró en las comunidades autónomas de Andalucía, Cataluña, Madrid, Comunidad Valenciana, Islas Baleares e Islas Canarias que aglutinaron el 62,1\% de las respuestas. Estas comunidades coinciden, por un lado, con las comunidades autónomas donde se sitúan la mayor parte de los destinos turísticos españoles objeto de análisis y, por otro, también con las que, históricamente, reciben más llegadas de turistas internacionales (INE, 2017a).

\section{RESULTADOS}

\subsection{Percepción de los residentes sobre los impactos turísticos en los destinos turísticos}

De los once ítems analizados, el que muestra una media con un valor más alto es el de "El turismo supone mayores oportunidades de empleo para los residentes e inversión para negocios locales" $(\mathrm{M}=4,05, \mathrm{DT}=1,061)$ seguido de "El turismo contribuye al aumento del número de actividades de ocio y culturales" $(\mathrm{M}=3,87, \mathrm{DT}=1,056)$ y "El turismo contribuye a la creación y/o mejora en las infraestructuras y servicios públicos” (M=3,73, DT=1,098).

\section{Tabla 5 \\ VALORACIÓN DE LAS VARIABLES DE PERCEPCIÓN DE LOS IMPACTOS DEL TURISMO EN EL MUNICIPIO}

\begin{tabular}{llcc}
\hline & & Media & DT \\
\hline & Contribución al empleo & 4,05 & 1,061 \\
& Contribución a las actividades de ocio y culturales & 3,87 & 1,056 \\
$\begin{array}{l}\text { Impactos } \\
\text { positivos }\end{array}$ & Contribución a infraestructuras & 3,73 & 1,098 \\
& Contribución a la conservación del patrimonio & 3,71 & 1,116 \\
& Contribución al intercambio cultural & 3,51 & 1,162 \\
& Contribución a la identidad cultural local & 3,44 & 1,176 \\
\hline
\end{tabular}




\begin{tabular}{llcc}
\hline & & Media & DT \\
\hline & Incremento de los precios & 3,28 & 1,169 \\
& Masificación en playas, parques, áreas recreativas, etc. & 3,16 & 1,266 \\
$\begin{array}{l}\text { Impactos } \\
\text { negativos }\end{array}$ & Aumento del ruido, polución, tráfico o suciedad & 2,98 & 1,233 \\
& Daños en el medioambiente & 2,73 & 1,201 \\
& Destrucción patrimonio cultural & 2,13 & 1,086 \\
\hline
\end{tabular}

$\mathrm{N}=1.027$

Fuente: Elaboración propia.

Por el contrario, las variables que presentan valores más bajos son la afirmación "El turismo contribuye a la destrucción del patrimonio cultural" $(\mathrm{M}=2,13$, DT=1,086) y la variable "El turismo produce daños en el medio ambiente" (M=2,73, DT=1,201).

En este sentido, resulta interesante destacar que los ítems mejor valorados son ítems relacionados con impactos positivos generados por el turismo y que, por el contrario, los que presentan valores medios más bajos, es decir, con los que los encuestados se encuentran menos de acuerdo, están relacionados con los impactos negativos.

Por otro lado, de los resultados obtenidos cabe destacar también el alto valor de las desviaciones típicas en ítems como "la masificación en playas, parques, áreas recreativas, etc." (DT=1,266), "el aumento del ruido, polución, tráfico o suciedad" (DT=1,233) o "los daños en el medioambiente" (DT=1,201). Estas desviaciones muestran una alta dispersión de las respuestas, quizá debida a la existencia de diversos perfiles de personas que perciben los impactos negativos de manera muy diferente.

\subsection{Percepción de los residentes sobre los impactos turísticos en destinos de sol y playa frente a los destinos de interior}

Como observamos en la revisión de la literatura académica, una de las variables que influye en la percepción de los impactos por parte de la población son las características de los destinos turísticos.

Para plantear nuestra investigación, hemos considerado que dentro de las características propias del destino la tipología desarrollada en el mismo podría resultar importante a la hora de explicar las diferencias entre las percepciones de unos y otros residentes. Así, en nuestro caso hemos hecho una distinción entre las comunidades autónomas en las que predomina el turismo de sol y playa y las de turismo de interior. Tal y como se observa en la tabla 6 se aprecian diferencias en todas las medias al comparar los dos tipos de destinos si bien, estas diferencias no son significativas en todos los casos. 


\section{Tabla 6}

Diferencias registradas en la percepción de los impactos en el destino en función de si pertenecen a una comunidad de sol y playa o de interior

\begin{tabular}{|c|c|c|c|c|c|c|c|}
\hline & & & $\mathbf{N}$ & Media & DT & $\mathbf{F}$ & GI \\
\hline \multirow{12}{*}{$\begin{array}{l}\text { Impactos } \\
\text { positivos }\end{array}$} & \multirow{2}{*}{$\begin{array}{l}\text { Contribución al } \\
\text { empleo }\end{array}$} & $\begin{array}{l}\text { Sol y } \\
\text { playa }\end{array}$ & 604 & 4,08 & 1,045 & 1,481 & 1,1025 \\
\hline & & Interior & 423 & 4,00 & 1,083 & & \\
\hline & \multirow{2}{*}{$\begin{array}{l}\text { Contribución a } \\
\text { las actividades } \\
\text { de ocio y } \\
\text { culturales }\end{array}$} & $\begin{array}{l}\text { Sol y } \\
\text { playa }\end{array}$ & 604 & 3,83 & 1,047 & 2,590 & 1,1025 \\
\hline & & Interior & 423 & 3,93 & 1,067 & & \\
\hline & \multirow{2}{*}{$\begin{array}{l}\text { Contribución a } \\
\text { infraestructuras }\end{array}$} & $\begin{array}{l}\begin{array}{l}\text { Sol y } \\
\text { playa }\end{array} \\
\end{array}$ & 604 & 3,75 & 1,079 & ,926 & 1,1025 \\
\hline & & Interior & 423 & 3,69 & 1,126 & & \\
\hline & \multirow{2}{*}{$\begin{array}{l}\text { Contribución a } \\
\text { la conservación } \\
\text { del patrimonio }\end{array}$} & $\begin{array}{l}\text { Sol y } \\
\text { playa }\end{array}$ & 604 & 3,60 & 1,110 & *12,939 & 1,1025 \\
\hline & & Interior & 423 & 3,86 & 1,110 & & \\
\hline & \multirow{2}{*}{$\begin{array}{l}\text { Contribución } \\
\text { al intercambio } \\
\text { social }\end{array}$} & $\begin{array}{l}\text { Sol y } \\
\text { playa }\end{array}$ & 604 & 3,42 & 1,168 & $* 8,827$ & 1,1025 \\
\hline & & Interior & 423 & 3,63 & 1,142 & & \\
\hline & \multirow{2}{*}{$\begin{array}{l}\text { Contribución } \\
\text { a la identidad } \\
\text { cultural local }\end{array}$} & $\begin{array}{l}\text { Sol y } \\
\text { playa }\end{array}$ & 604 & 3,28 & 1,155 & $* 26,120$ & 1,1025 \\
\hline & & Interior & 423 & 3,66 & 1,171 & & \\
\hline \multirow{10}{*}{$\begin{array}{l}\text { Impactos } \\
\text { negativos }\end{array}$} & \multirow{2}{*}{$\begin{array}{l}\text { Incremento de } \\
\text { los precios }\end{array}$} & $\begin{array}{l}\text { Sol y } \\
\text { playa }\end{array}$ & 604 & 3,36 & 1,158 & $* 5,682$ & 1,1025 \\
\hline & & Interior & 423 & 3,18 & 1,179 & & \\
\hline & \multirow{2}{*}{$\begin{array}{l}\text { Masificación } \\
\text { en playas, } \\
\text { parques, áreas } \\
\text { recreativas, etc. }\end{array}$} & $\begin{array}{l}\begin{array}{l}\text { Sol y } \\
\text { playa }\end{array} \\
\end{array}$ & 604 & 3,39 & 1,220 & $* 52,324$ & 1,1025 \\
\hline & & Interior & 423 & 2,83 & 1,257 & & \\
\hline & \multirow{2}{*}{$\begin{array}{l}\text { Aumento del } \\
\text { ruido, polución, } \\
\text { tráfico o } \\
\text { suciedad }\end{array}$} & $\begin{array}{l}\text { Sol y } \\
\text { playa }\end{array}$ & 604 & 3,13 & 1,249 & $* 22,962$ & 1,1025 \\
\hline & & Interior & 423 & 2,76 & 1,177 & & \\
\hline & \multirow{2}{*}{$\begin{array}{l}\text { Daños en el } \\
\text { medioambiente }\end{array}$} & $\begin{array}{l}\text { Sol y } \\
\text { playa }\end{array}$ & 604 & 2,85 & 1,217 & $* 16,109$ & 1,1025 \\
\hline & & Interior & 423 & 2,55 & 1,157 & & \\
\hline & \multirow{2}{*}{$\begin{array}{l}\text { Destrucción } \\
\text { patrimonio } \\
\text { cultural** }\end{array}$} & $\begin{array}{l}\begin{array}{l}\text { Sol y } \\
\text { playa }\end{array} \\
\end{array}$ & 604 & 2,21 & 1,108 & $* 8,225$ & 1,1025 \\
\hline & & Interior & 423 & 2,01 & 1,045 & & \\
\hline
\end{tabular}

Nota: Homogeneidad de varianzas Levene $\mathrm{p}>0.05$ excepto $* *$ que se acepta al $10 \%$. $* \mathrm{p}<0,05$ 
En cuanto a los impactos positivos, existen diferencias significativas entre los destinos de interior y los de sol y playa en la percepción que los grupos de interés tienen acerca de la "contribución del turismo a la identidad local" F 26,120, p=.00 siendo la percepción de que el turismo favorece la consolidación de la identidad local mayor en los destinos de interior $(3,66$, DT 1,171$)$ que en los destinos de sol y playa $(3,28$ DT 1,155$)$. Muy relacionada con esta variable está la "contribución a la conservación del patrimonio" F $12,939 \mathrm{p}=.00$ que se comporta de forma muy parecida a la anterior pues se percibe de forma más positiva en las comunidades de interior $(3,86$, DT 1,11$)$ que en las de sol y playa $(3,60$, DT 1,11). En esta línea, observamos también que la variable "contribución al intercambio social" F 8,827 p=.00 es percibida de forma más negativa en los destinos de sol y playa $(3,42$, DT 1,168$)$ que en los destinos de interior $(3,63$, DT 1,142$)$. Se puede decir por tanto que los impactos positivos de tipo sociocultural son mejor percibidos en los destinos de interior que en los de sol y playa.

No hay diferencias significativas en cambio entre los residentes que habitan en comunidades de turismo sol y playa y comunidades de turismo de interior en la percepción de que el turismo contribuye a "la creación de infraestructuras" F 0,926, p=.33, a "la creación de empleo" F 1,481, p=.22 y al "fomento de actividades de ocio y culturales" 2,590, p=.10.

En cuanto a los impactos negativos, observamos que existen diferencias significativas entre los grupos de interés de destinos de interior respecto a los de sol y playa en varias de las variables estudiadas. Es el caso de la percepción sobre el "aumento del ruido, polución, tráfico o suciedad" F 22,962, p=.00 donde es más elevada la percepción en destinos de sol y playa $(3,13$, DT 1,249$)$ que en destinos de interior $(2,76$, DT 1,177$)$. Ocurre algo similar con las variables "Incremento de los precios" $\mathrm{F} 5,682, \mathrm{p}=.01$ siendo más sensibles al proceso inflacionario en los destinos de sol y playa $(3,36$, DT 1,158$)$ que en los destinos de interior $(3,18$, DT 1,179) y "daños en el medio ambiente" F 16,109, p=.00 en la que también existe una percepción de los impactos medioambientales más negativa en los destinos de sol y playa $(2,85$, DT 1,217$)$ que en los de interior $(2,55$ DT 1,157$)$.

Pero sin duda en la variable donde encontramos más diferencias es en la percepción de “masificación en playas, parques, áreas recreativas, etc.” F 52,324, p=.00 siendo percibida con una media superior a 3 en los destinos de sol y playa $(3,39$, DT 1,220$)$ e inferior a 3 en los de interior $(2,83$, DT 1,257$)$.

Así pues, podemos afirmar que la percepción de los impactos negativos generados por el turismo es superior en los destinos de sol y playa que en los de interior, teniendo los primeros una imagen más negativa del desarrollo turístico.

\section{DISCUSIÓN}

Los resultados de la investigación muestran que los ítems mejor valorados son aquellos relacionados con impactos positivos generados por el turismo, mientras que los que presentan valores medios más bajos están relacionados con los impactos negativos. Estos resultados son similares a los de otros estudios acerca de la percepción de los impactos del turismo por parte de los grupos de interés donde se afirma que, generalmente, los residentes perciben los impactos generados por el turismo de forma positiva (Liu y Var, 
1986; Besculides et al., 2002; Gursoy et al., 2002; Sharma y Dyer, 2009; Cardona, 2012; Markovic y Klaric, 2015).

Algunos autores atribuyen esta percepción positiva al peso que tienen los impactos económicos positivos, como el crecimiento económico o la generación de empleo (Var y Kim, 1990; Tosun, 2002; Vargas-Sánchez et al., 2011; Cardona, 2012) especialmente en situaciones económicas de crisis (Besculides et al., 2002; Markovic y Klaric, 2015; Garau-Vadell et al., 2018). Precisamente, nuestra investigación coincide con estos estudios al demostrar que la generación de empleo es el impacto mejor valorado por los residentes, independientemente del destino.

Otros autores (Wang y Chen, 2015) otorgan un mayor peso a factores sociales como la identidad local, de tal modo que los residentes se mostrarán favorables al turismo si esta variable es valorada de forma positiva, tal y como ocurre en este estudio, dato que refuerza la tesis anteriormente expuesta.

En cuanto a los impactos nocivos generados por el turismo, los residentes estiman que aquel que genera un mayor daño es el incremento de los precios, tal y como muestran otros autores (Var y Kim, 1990; Vargas-Sánchez et al., 2011; Markovic y Klaric, 2015).

Teniendo en cuenta estos resultados obtenidos podemos validar solo parcialmente la $\mathrm{H}_{1}$ que hacía referencia a que existen diferencias significativas en la percepción que los residentes tienen de los impactos positivos generados por el turismo en los destinos españoles de sol y playa y los de interior. A este respecto, los resultados muestran que, si bien existen diferencias significativas en cuanto al impacto del turismo en la conservación del patrimonio, la contribución al intercambio social o a la identidad local, no ocurre lo mismo con la contribución al empleo, contribución a las actividades de ocio y culturales y contribución a la creación de infraestructuras.

En cambio, podemos aceptar $\mathrm{H}_{2}$ porque se han identificado diferencias significativas en la percepción de los impactos negativos del turismo en función del tipo de destino.

Respecto a nuestra hipótesis $\mathrm{H}_{3}$ que hacía referencia a que los residentes de destinos turísticos de interior perciben de forma más intensa los impactos positivos generados por el turismo, podemos validarla parcialmente. En concreto, los residentes de comunidades de interior muestran una percepción más positiva de los impactos socioculturales del turismo, al igual que ocurría en el estudio Sharma y Dyer (2009). Merecen especial atención las variables relacionadas con la conservación del patrimonio y la preservación de la identidad cultural, percibidas de manera más positiva en los destinos de interior, tal vez por el tipo de turismo que se suele desarrollar en esas comunidades, predominantemente de tipo cultural o rural.

Por último, podemos aceptar $\mathrm{H}_{4}$ pues efectivamente los residentes de destinos turísticos de sol y playa perciben de forma más intensa los impactos negativos generados por el turismo. Los resultados muestran que los impactos nocivos del turismo, sobre todo aquellos relacionados con el medioambiente, tales como la masificación y la contaminación, se perciben de forma más negativa en los destinos de sol y playa, respaldando la tesis de autores como Rodríguez (1994) o Zahedi (2008). Asimismo, tal y como ocurre en la investigación de Markovic y Klaric (2015) el proceso inflacionario se percibe más en las comunidades de sol y playa que en aquellas de interior. 


\section{CONCLUSIONES}

La medición de los impactos generados por el sector turístico se ha convertido en uno de los temas recurrentes en el ámbito de investigación de la planificación y gestión de los destinos turísticos. En este sentido, a la hora de identificar y medir los impactos turísticos, se considera que la percepción que tienen los residentes de estos impactos es tanto o más importante que los impactos en sí mismos.

En concreto, podemos afirmar que los diversos residentes de los destinos turísticos españoles tienen una visión positiva del desarrollo turístico en sus municipios, estando más de acuerdo con las afirmaciones relacionadas con los impactos positivos, especialmente con la contribución del turismo a la generación de empleo y a las actividades de ocio y culturales.

Por el contrario, en cuanto a la percepción que muestran las partes interesadas de los destinos españoles acerca de los impactos negativos generados por el sector turístico, los resultados muestran que existe menos conformidad con los ítems relacionados con los impactos nocivos, es decir, se valoran más positivamente los impactos positivos mientras los negativos no afectan tanto a su percepción sobre el desarrollo turístico. Únicamente, el incremento de precios es un aspecto que se valora negativamente.

En esta línea, resulta significativo el hecho de que las afirmaciones con valores más altos tanto en el grupo de impactos positivos como en el de impactos negativos estén relacionadas con el ámbito económico.

Al analizar si existen diferencias en las percepciones de los residentes en función del tipo de destino en el que residen, se ha podido comprobar que estas diferencias existen ya que los residentes de los destinos de sol y playa perciben de forma más negativa el turismo que aquellos que residen en los destinos de interior. En concreto, son las variables relacionadas con los impactos ambientales negativos del turismo las que se perciben más en los destinos de sol y playa. Aquí debemos mencionar la masificación como una de las problemáticas más importantes del turismo de sol y playa. Por su parte, los residentes en destinos de interior perciben de manera más intensa los impactos especialmente los impactos socioculturales positivos, sobre todo aquellos relacionados con la conservación del patrimonio, la contribución al intercambio social y la preservación de la identidad cultural.

En definitiva, los resultados de este estudio suponen un importante avance en el conocimiento de la percepción que del turismo tienen los residentes de los destinos turísticos españoles dado que el ámbito de estudio de la investigación es el total nacional constatándose la percepción positiva del turismo que hay en el país. Este optimismo se fundamenta especialmente en los impactos económicos positivos que el turismo genera en nuestros destinos.

Asimismo, el estudio supone una aportación importante ya que permite constatar que existen diferencias en la percepción de los impactos turísticos en función de la tipología de destino turístico. En concreto, se constata que la percepción que tienen los residentes de los destinos de sol y playa es más negativa que en los destinos de interior, siendo los impactos nocivos sobre el medio ambiente los que peor se perciben.

Con el fin de continuar profundizando en el estudio de la percepción de los impactos generados por el turismo en los destinos y en concreto en España, sería interesante que en 
futuros estudios se analizara también la influencia sobre la percepción de variables como la influencia de las variables sociodemográficas, el grado de contacto con los turistas o la situación económica del destino.

\section{REFERENCIAS}

AGUILÓ, E. y ROSELLÓ, J. (2005): «Host community perceptions: a cluster analysis», Annals of Tourism Research, 32 (4), pp. 925-941.

AGUILÓ, E., BARROS, V., GARCÍA, M.A., y ROSELLÓ, J. (2004): Las actitudes de los residentes en Baleares frente al turismo. Palma, Universidad de las Islas Baleares.

AKIS, S., PERISTIANIS, N. y WARNER, J. (1996): «Residents' Attitudes to Tourism Development: The Case of Cyprus», Tourism Management, 17 (7), pp. 481-494.

ALMEIDA-GARCÍA, F., PELÁEZ-FERNÁNDEZ, M.A., BALBUENA-VÁZQUEZ, A. y CORTÉS-MACIAS, R. (2016): «Residents' perceptions of tourism development in Benalmadena (Spain)», Tourism Management, 54 (3), pp. 259-274.

ANDERECK, K. y NYAUPANE, G. (2011): «Exploring the nature of tourism and quality of life perceptions among residents», Journal of Travel Research, 50 (3), pp. 248-260.

ANDERECK, K., VALENTINE, K, VOGT, C. y KNOPF, R. (2005): «A cross-cultural analysis of tourism and quality of life perceptions», Journal of Sustainable Tourism, 15 (5), pp. 483-502.

AP, J. (1992): «Residents perceptions on tourism impacts», Annals of Tourism Research, 19 (4), pp. 665-690.

BESCULIDES, A., LEE, M.E. y McCORMICK, P.J. (2002): «Resident's perceptions of the cultural benefits of tourism», Annals of Tourism Research, 29 (2), pp. 303-319.

BESTARD, B. y NADAL, R. (2007): «Attitudes toward tourism and tourism congestion», Région et Développment, 25, pp. 193-207.

BUJOSA, A. y ROSELLÓ, J. (2007): «Modelling environmental attitudes toward tourism», Tourism Management, 28 (3), pp. 688-695.

BUTLER, R. (1980): «The concept of a tourism area cycle of evolution», Canadian Geographer, 24 (1), pp. 5-12.

CARDONA, J.R. (2012): Actitudes de los residentes hacia el turismo en destinos turísticos consolidados: el caso de Ibiza. Tesis doctoral, Palma: Universidad Illes Baleares.

CHOI, H.C. y MURRAY, I. (2010): «Resident attitudes towards sustainable community tourism», Journal of Sustainable Tourism, 18 (4), pp. 575-594.

COCCOSIS, H. y MEXA, A. (2002): Defining, measuring and evaluating carrying capacity in European tourism destinations. B4-3040/2000/294577/MAR/D2. Athens: Environmental Planning Laboratory by the European Commission, DirectorateGeneral for Environment, Nuclear Safety and Civil Protection. 
COHEN, E. (1978): «The impact of tourism on the physical environment», Annals of Tourism Research, 5 (2), pp. 215-37.

DANIELS, M.J. (2007): «Central place theory and sport tourism impacts», Annals of Tourism Research, 34 (2), pp. 332-347.

DEERY, M., JAGO, L. y FREDLINE, L. (2012): «Rethinking social impacts of tourism research: a new research agenda», Tourism Management, 33 (1), pp. 64-73.

DÍAZ, R. y GUTIÉRREZ, D. (2010): «La actitud del residente en el destino turístico de Tenerife: evaluación y tendencia», PASOS. Revista de Turismo y Patrimonio Cultural, 8(4), pp. 431-444.

DIEDRICH, A. y GARCÍA, E. (2009): «Local perceptions of tourism as indicators of destination decline», Tourism Management, 30 (4), pp. 512521.

DOXEY, G. (1975): A causation theory of visitor-resident irritants: methodology and research inferences in the impact of tourism. Sixth Annual Conference Proceedings of the Travel Research Association. San Diego (California), pp. 195-198.

DYER, P., GURSOY, D., SHARMA, B. y CARTER, J. (2007): «Structural modelling of resident perceptions of tourism and associated development on the Sunshine Coast, Australia», Tourism Management, 28 (2), pp. 409-422.

EASTERLING, D. (2004): «The residents' perspective in tourism research: a review and synthesis», Journal of Travel \& Tourism Marketing, 17(4), pp. 45-62.

FAULKNER, B. y TIDESWELL, C. (1997): «A framework for monitoring community impacts of tourism», Journal of Sustainable Tourism, 5(1), pp. 3-28.

FIGUEROLA PALOMO, M. (2000): Introducción al estudio económico del turismo. Madrid: Civitas.

FIGUEROLA PALOMO, M., FERNÁNDEZ MUÑOZ, J.J., GÓMEZ BRUNA, D. y MARTÍN DUQUE, C. (2015) Medición del impacto del turismo en la economía, el empleo y el medio ambiente en el ámbito local. Madrid: EOI.

FREDLINE, E. y FAULKNER, B. (2000): «Host community reactions: a cluster analysis», Annals of Tourism Research, 27 (3), pp. 763-784.

GARAU-VADELL, J.B., GUTIÉRREZ-TAÑO, D. y DÍAZ-ARMAS, R. (2018): «Economic crisis and residents' perception of the impacts of tourism in mass tourism destinations», Journal of Destination Marketing \& Management, 7, pp. 68-75.

GRABURN, N.H. (1976): Ethics and Tourist Arts: Cultural Expressions from the Fourth World. Berckley: University of California Press.

GURSOY, D. y RUTHERFORD, D.G. (2004): «Host attitudes toward tourism: An improved structural model», Annals of Tourism Research, 31(3), pp. 495-516.

GURSOY, D., CHI, C.G. y DYER, P. (2010): «Local's attitudes toward mass and alternative tourism: The case of Sunshine Coast, Australia», Journal of Travel Research, 49, pp. 381-394. 
GURSOY, D., JUROWSKI, C. y UYSAL, M. (2002): «Resident attitudes: A structural modeling approach», Annals of Tourism Research, 29(1), pp. 79-105.

HARALAMBOPOULOS, N. y PIZAM, A. (1996): «Perceived impacts of tourism: The case of Samos», Annals of Tourism Research, 23 (3), pp. 503-526.

HERNÁNDEZ MARTÍN, R. (2004): «Impacto económico del turismo. El papel de las importaciones como fugas del modelo», Revista de economía, 817, pp. 23-34.

HERNÁNDEZ, R., FERNÁNDEZ, C. y BAPTISTA, P. (2010): Metodología de la investigación. México: McGraw Hill.

HOLDEN, A. (2009): «An Introduction to Tourism-Environment Relationships», en Ecotourism and Environmental Sustainability Principles and Practice, Surrey: Ashgate, pp. 17-30.

HUETE, R. (2010): «Opiniones y actitudes ante el turismo residencial en el sur de la Comunidad Valenciana», Pasos. Revista de Turismo y Patrimonio Cultural, 8(4), pp. 445-461.

INE (2017a): Estadística de movimientos turísticos en frontera. Frontur. Disponible en: http://www.ine.es/dyngs/INEbase/es/operacion.htm?c=Estadistica_C\&cid=1254736 $176996 \&$ menu $=$ ultiDatos\&idp $=1254735576863$.

INE (2017b): Encuesta de gasto turístico. Egatur. Disponible en: http://www.ine.es/ dyngs/INEbase/es/operacion.htm?c=Estadistica_C\&cid $=1254736177002 \& \mathrm{menu}=\mathrm{ul}$ tiDatos\&idp $=1254735576863$.

IVARS BAIDAL, J. (2001): Planificación y gestión del desarrollo turístico sostenible: propuestas para la creación de un sistema de indicadores. Proyecto METASIG. Alicante: Universidad de Alicante.

JOHNSON, J., SNEPENGER, D. y AKIS, S. (1994): «Residents' perceptions of tourism development», Annals of Tourism Research, 21 (3), pp. 629-642.

JUROWSKI, C. y GURSOY, D. (2004): «Distance effects on residents' attitudes toward tourism», Annals of Tourism Research, 31 (2), pp. 296-312.

KING, B., PIZAM, A. y MILMAN, A. (1993): «Social impacts of tourism: host perceptions», Annals of Tourism Research, 20 (4), pp. 650-665.

LANKFORD, V. (1994): «Attitudes and perceptions toward tourism and rural regional development», Journal of Travel Research, 32 (2), pp. 35-43.

LÁZARO QUINTERO, J. (2004): «Los impactos económicos, socioculturales y medioambientales del turismo y sus vínculos con el turismo sostenible», Anales del Museo de América, 12, pp. 263-274.

LIBURD, J. y EDWARDS, D. (2010): Understanding the Sustainable Development of Tourism. Oxford: Goodfellow Publishers ltd.

LINDBERG, K. y JOHNSON, R. L. (1997): «Modelling resident attitudes toward tourism», Annals of Tourism Research, 24 (2), pp. 402-424. 
LIU, J. y VAR, T. (1986): «Resident Attitudes Toward Tourism Impacts in Hawaii», Annals of Tourism Research, 13 (2), pp. 193-214.

LIU, J., SHELDON, P.J. y VAR, T. (1987): «Resident perception of the environmental impacts of tourism», Annals of Tourism Research, 14 (1), pp. 17-37.

LONG, P.T., PERDUE, R.R. y ALLEN, L.R. (1990): «Rural Resident Tourism Perceptions and Attitudes by Commumty Level of Tourism», Journal of Travel Research, 28(3), pp. 3-9.

MADRIGAL, R. (1995): «Residents' Perceptions and the Role of Government», Annals of Tourism Research, 22 (1), pp. 86-102.

MARKOVIC, I. y KLARIC, Z. (2015): «Attitudes of local population of tourism impacts on destination sustainability: Case of Croatia», Turizam, 19 (3), 98-110.

MARRERO, J.R. (2006): «El discurso de rechazo al turismo en Canarias: una aproximación cualitativa», PASOS. Revista de Turismo y Patrimonio Cultural, 4(3), pp. 327- 341 .

MASON, P. (2003): Tourism Impacts, Planing and Management. Oxford: Elseiver Butterworth Heinemann.

MASON, P. (2009): «La gestión de los impactos socioculturales del turismo» en Problemas específicos de gestión en las diversas empresas turísticas, Madrid: Síntesis, pp. 67-87.

McGEHEE, N. y ANDEREK, K. (2004): «Factors predicting rural residents' support for tourism», Journal of Travel Research, 43 (2), pp. 131-140.

OCDE (2002): OECD programme on sustainable consumption. Sector case studies series. Household tourism travel: trends, environmental impacts and policy responses. ENV/EPOC/WPNEP (2001)14/FINAL. Paris: OCDE.

OCDE (2010): Glosario de los principales términos sobre evaluación y gestión basada en resultados. París: OCDE.

OMT (2005). Indicadores de desarrollo sostenible para los destinos turísticos. Guía práctica. Madrid: OMT.

OVIEDO, M.A., CASTELlANOS, M. y MARTíN, D. (2008): «Gaining residents' support for tourism and planning», International Journal of Tourism Research, 10 (2), pp. 95-109.

PEARCE, J. A. (1980): «Host Community Acceptance of Foreign Tourism», Annals of Tourism Research, 7 (2), pp. 224-233.

PEREIRO PÉREZ, X. y DE LEÓN SMITH, C. (2007): Los impactos del turismo en Kuna Yala. Madrid: Ramón Areces.

PÉREZ, E. y NADAL, J. (2005): «Host community perceptions: a cluster analysis», Annals of Tourism Research, 32(4), pp. 925-941. 
PÉREZ, M. (2004): Manual Del Turismo Sostenible: Cómo Conseguir Un Turismo Social, Económico y Ambientalmente Responsable. Madrid: Mundi-Prensa Libros.

PIZAM, A. (1978): «Tourism impacts: the social costs to the destination as perceived by its residents», Journal of Travel Research, 16 (4), pp. 8-12.

PULIDO, J.I. (2004): «El medio ambiente en la política turística española», Quaderns de Política Econòmica, 7, pp. 94-114.

RASOOLIMANESH, S.M, RINGLE, C.M., JAAFAR, M. y RAMAYAH, T. (2017): «Urban vs. rural destinations: Residents' perceptions, community participation and support for tourism development», Tourism Management, 60 (1), pp. 147-158.

RITCHIE, J.R.B. (1988): «Consensus policy formulation in tourism: measuring resident views via survey research», Tourism Management, 9 (3), pp. 199-212.

RODRÍGUEZ, F. (1994): «El impacto ambiental del turismo» en Desarrollo regional y crisis del turismo en Andalucía. Actas del simposio hispano-francés-Almería, pp. 331-338.

ROYO, M. y RUIZ, M. E. (2009): «Actitud del residente hacia el turismo y el visitante: factores determinantes en el turismo y excursionismo rural-cultural», Cuadernos de Turismo, 23, pp. 217-236.

SAARIEN, J. (2006): «Traditions of sustainability in tourism studies», Annals of Tourism Research, 33(4), pp. 1121-1140.

SANCHO, A. (1998). Introducción al turismo. Madrid: OMT.

SANCHO, A. y GARCÍA, G. (2006): «¿Qué indica un indicador? Análisis comparativo en los destinos turísticos», Revista de Análisis Turístico, 2, pp. 69-85.

SANCHO, A., GARCÍA, G. y ROZO, E. (2007): «Comparativa de indicadores de sostenibilidad para destinos desarrollados, en desarrollo y con poblaciones vulnerables», Annals of tourism research en español, 9 (1), pp. 150-177.

SHARMA, B. y DYER, P. (2009): «An investigation of differences in residents' perceptions on the Sunshine Coast: tourism impacts and demographic variables», Tourism Geographies, 11(2), pp. 187-213.

SHARPLEY, R. (2014): «Host perceptions of tourism: A review of the research», Tourism Management, 42, pp. 37-49.

SHELDON, P. y VAR, T. (1984): «Residents attitudes toward tourism in North Wales», Tourism Management, 5 (1), pp, 40-47.

SMITH, V. (1977): Host and Gest: An Anthropology of Tourism. Philadephia: University of Pensylvania Press.

SOARES, J. C., GANDARA, J. M. e IVARS, J. (2012): «Indicadores para analizar la evolución del ciclo de vida de los destinos turísticos litorales», Investigaciones Turísticas, 3, pp. 19-38.

TEYE, V., SÖNMEZ, S. y SIRAKAYA, E. (2002): «Residents' attitudes toward tourism development», Annals of Tourism Research, 29 (3), pp. 668-688. 
THOMASON, P., CROMPTON, J. y KAMP, B. (1979): «A study of the attitudes of impacted groups within a host community toward prolonged stay tourist visitors», Journal of Travel Research, 17(2), pp. 2-6.

TOSUN, C. (2002): «Host perceptions of impacts. A comparative tourism study», Annals of Tourism Research, 29 (1), pp. 231-264.

TRIBE, J. (2009): El impacto económico del turismo» en Problemas específicos de gestión en las diversas empresas turísticas. Madrid: Síntesis, pp. 51-66.

TURNER, L. y ASH, J. (1975): The Golden Hordes: International Tourism and the Pleasure Periphery. London: Constable.

VAR, T. y KIM, Y. (1990): «Tourism impact and its control: A review and synthesis of 20 years of research» en Quality of life Studies in Marketing and Management, WA: Virginia Tech. Center for Strategy and Marketing Studies, pp. 292-302.

VARGAS-SÁNCHEZ, A., PLAZA-MEJÍA, M. y PORRAS-BUENO, N. (2009): «Understanding residents' attitudes toward the development of industrial tourism in a former mining community», Journal of Travel Research, 47 (3), pp. 373-387.

VARGAS-SÁNCHEZ, A., PORRAS-BUENO, N. y PLAZA-MEJÍA, M.A. (2011): «Explaining residents' attitudes to tourism. Is a universal model possible?», Annals of Tourism Research, 38 (2), pp. 460-480.

VÁZQUEZ VARELA, C. y MARTÍN GIL, F. (2011): «Problemas de sostenibilidad del turismo rural en España», Anales de Geografía, 31 (1), pp. 171-194.

WALL, M. y MATHIESON, A. (2006): Tourism: change, impacts and opportunities. England, Pearson. Prentice Hall.

WANG, S. y CHEN, J.S. (2015): «The influence of place identity on perceived tourism impacts», Annals of Tourism Research, 52 (1), pp. 16-28.

WILLIAMS, J. y LAWSON, R. (2001): «Community Issues and Resident Opinions of Tourism», Annals of Tourism Research, 28 (2), pp. 269-290.

WORLD TRAVEL \& TOURISM COUNCIL (2013). Travel \& Tourism Economic Impact 2013. UK: World Travel \& Tourism Council.

WU, C. (2009): «Sustainable Development Conceptual Framework in Tourism Industry Context in Taiwan: Resource Based View», Conference of the International Journal of Arts and Sciences, 2 (1), pp. 1-11.

YOON, Y., GURSOY, D. y CHEN, J.S. (2001): «Validating a tourism development theory with structure equation modeling», Tourism Management, 22 (4), pp. 363-372.

YOUNG, G. (1973): Tourism: blessing or blight? Harmondsworth: Penguin.

ZAHEDI, S. (2008): «Tourism impact on coastal environment. Environmental Problems in Coastal Regions» en VII. WIT Transactions on The Built Environment, 99, pp. 45-57. 
